\title{
MODERN MODELS AND APPROACHES FOR DESIGN OF ARCHITECTURE OF A SOFTWARE APPLICATION FOR MONITORING AND QUALITY ASSESSMENT IN HIGHER EDUCATION
}

\author{
Kristina Kilova, ${ }^{1}$ Vanya Lazarova, ${ }^{2}$ Tanya Kitova, ${ }^{3}$ \\ Desislava Bakova, ${ }^{4}$ Angelina Kirkova-Bogdanova ${ }^{5}$
}

\begin{abstract}
Processes such as economic and cultural globalization put forward the issue on the quality of education. The increasing interconnectivity between economy and different societal spheres, driven by the accelerated development of information technology and the free movement of capital, increasingly links the prosperity of a country and its individuals to knowledge and technology, to the ability to learn and understand experience. In this context, it is of paramount importance to set national educational goals and to shape relevant policies.

Constructive student feedback, establishment of systems of continuous control, and implementation of changes based on a scientific conceptual framework will help the education to move from quality assurance to quality improvement. The quality of education is a responsibility of all participants - lecturers, students, institution management. Automating the feedback process with students will lead to a faster analysis of the results, making adequate management decisions in order to achieve the ultimate goal - improving the quality of higher education.

In the present paper the need for regularly providing students with the opportunity actively to participate in the management of quality of education is justified. The paper deals with the automation of collection, analysis of the needed information and consequent adequate management decisions. A conceptual model of "Web-based information system for quality assessment of the education" in higher schools with a focus on the survey and its design are also presented.
\end{abstract}

UDC Classification: 378; DOI: http://dx.doi.org/10.12955/cbup.v5.1000

Keywords: quality assessment, higher education, conceptual model, information system

\section{Introduction}

Quality and its assessment are essential for the modern management of education. It is included in the mission, goals, and tasks of every higher school detailing in the collection, analysis of the needed information and consequent adequate management decisions (Peeva, 2010).

The paper deals with the automation of these processes, including the analysis of the requirements for the development of a model of a "Web-based information system for quality assessment of education" (Web-SAQE) and its design. In the focus of the statement, we present modern models and approaches for a design of the architecture of the software application for monitoring and quality assessment in higher education. Functional and non-functional requirements and restrictions for the system are defined. The Automated Feedback Generator, for example, is an automated feedback software system, designed to provide superior quality assurance and efficiency in both assessing student assignments and providing feedback (Debuse et al., 2007). Testing has demonstrated that it offers quality control, efficiency, and effectiveness while generating consistent feedback from the student's perspective (Hadzhikoleva et al., 2010).

This paper presents the results of a study examining the major contemporary models and approaches for designing architecture of a software application and its construction. It provides a rationale for selected technology solutions for such a design and construction.

\section{Literature Review}

An analysis of the requirements for developing a model of the Web-Based System for Assessing the Quality of Education (Web-SAQE) revealed the following.

\footnotetext{
${ }^{1}$ Medical University - Plovdiv, Bulgaria, Faculty of Public Health, Dept. "Medical Informatics, Biostatistics and E-learning", k_kilova@abv.bg

${ }^{2}$ Plovdiv University "Paisii Hilendarski", Bulgaria, vanya@liveauthentic.net

${ }^{3}$ Medical University - Plovdiv, Bulgaria, Medical Faculty, Dept. "Anatomy, Histology and Embryology", tanyakitova@yahoo.com

${ }^{4}$ Medical University - Plovdiv, Bulgaria, Faculty of Public Health, Dept. "Healthcare Management", desislavabakova@abv.bg

${ }^{5}$ Medical University - Plovdiv, Bulgaria, Faculty of Public Health, Dept. "Medical Informatics, Biostatistics and E-learning", akirkova@meduniversity-plovdiv.bg
} 
Non-functional requirements, called quality requirements, are crucial in building a software system (Bontchev, 2012). The most significant of these are:

- Reliability, to fulfill the defined functionalities under certain conditions for a specified period;

- Multiple uses, the system modules must be reusable and unexpected, or invalid environmental conditions must be tolerated;

- Interoperability, the system must ensure compatibility with other products. It also must comply with established standards and norms for presenting information and technological realization (national and international);

- Scalability, the ability to add new functionalities without the need for major changes in the architecture;

- Sustainability, the designed software system must be resistant to damage;

- Easy maintenance, the system must be designed in a way that it requires easy maintenance;

- Modularity, the individual modules must be tested in isolation before being integrated into the overall system. They have to perform low connectivity and high consistency;

- Easy usability, the system must provide an interface which is easy to understand and use;

- Privacy and data security;

- Internet access including mobility; and

- Simultaneous access by multiple users.

The main restrictions are related to the data processing system, the realization of communication, consistency with copyright law, consistency with legal requirements for exchange of personal data, and access through the Internet (Lazarova, 2015; Doneva et al., 2011).

Thus, the defined requirements for a modern information system show a necessity for designing a Web-SAQE as an information system that provides quality management with the necessary level of customization. Hence, it requires a study of the following components:

- Environment; and

- Users (subjects).

The environment is the main component that contains services for assessing the quality of education, access to surveys, and control of user access to different functionalities of the system. It is represented by a technological platform that provides all necessary elements, technologies for synchronous and asynchronous communication, user management, content management, evaluation and monitoring, and web services.

\section{Data and Methodology}

For analyzing the requirements, a classification was created in which the functional requirements were placed into several groups, and quality requirements and restrictions to the system were identified. Target groups and their responsibilities and needs were classified.

The study examined the sites of national and international universities consistent with the educational policies and strategies for the development of higher education. This was prompted by a survey conducted in September 2016 that showed a positive attitude of students toward registration in a WebSAQE and in completing online feedback surveys as part of the quality management in the Medical University of Plovdiv (Kilova \& Stoyanova, 2016). As a result, this study defined functional requirements for a modern information system as follows:

- Maintenance of database about students, teachers, staff, curriculum, and data for suspended students;

- Automation of actions, such as:

- organizing and conducting surveys to assess the quality of education;

$\circ$ processing, analysis, and graphical presentation of data

- User management (roles, rights);

- Import, export data from or to various file formats; and

- Connecting with existing systems. 
The users were students, postgraduate students, teachers, and staff from the Medical University of Plovdiv.

An analysis of Web-SAQE design and conditions provided a description of the functional and quality requirements of the system, taking into account the basic subjects, objects, and processes in the field of research into the quality of education. All elements of the architecture of the software application were documented.

\section{Results and Discussion}

As a result of the studies and systematization of sources, consistent with the functional requirements formulated for the Web-SAQE, the following service groups were differentiated:

1. Administrative assistance to the process of the survey;

2. Process management of the surveys, i.e., feedback system of the medical university as part of the Web-SAQE;

3. User management (roles and rights); and

4. Security management and access to the system.

An essential feature of these service groups was their connection with each other and with external systems. Table 1 provides additional details on the management of the feedback system of the medical university.

\begin{tabular}{|l|c|c|}
\hline Table 1: Characteristics of actions of the Feedback System of the Medical University (FSMU) \\
\hline Automation of actions & $\begin{array}{c}\text { existing } \\
\text { survey } \\
\text { systems }\end{array}$ & FSMU \\
\hline $\begin{array}{l}\text { Connection with external systems and the ability to import-export } \\
\text { data in different file formats }\end{array}$ & $\sqrt{ }$ & $\sqrt{ }$ \\
\hline $\begin{array}{l}\text { Automatic processing of collecting general information about the } \\
\text { respondent. Integration with existing information system of the } \\
\text { medical university }\end{array}$ & - & $\sqrt{ }$ \\
\hline $\begin{array}{l}\text { Automatic control of the conduct of surveys } \\
\text { Automatic processing of surveys }\end{array}$ & - & $\sqrt{ }$ \\
\hline Archive data on students' opinion about the quality of education & - & \\
\hline Source: Author & & $\sqrt{ }$ \\
\hline
\end{tabular}

The automated actions listed in Table 1 were crucial in the design stage of the Web-SAQE. The study identified the following key management processes:

- Automating and creating surveys including defining the purpose and the respondents;

- Gathering information, including protection from unauthorized access and recurrence of responses from one person;

- Data processing, and disclosure and storage of the results; and

- Storing information about the history of voting.

An automated service of the survey process had the aim of automating the management including the administration of the following:

- Publicly available information;

- Planning the educational process; and

- Assessing and analyzing the results.

Figure 1 shows the integration of the Web-SAQE with external information systems of the Ministry of Education and Science (MES), the National Evaluation and Accreditation Agency (NEAA), and the Medical University. 


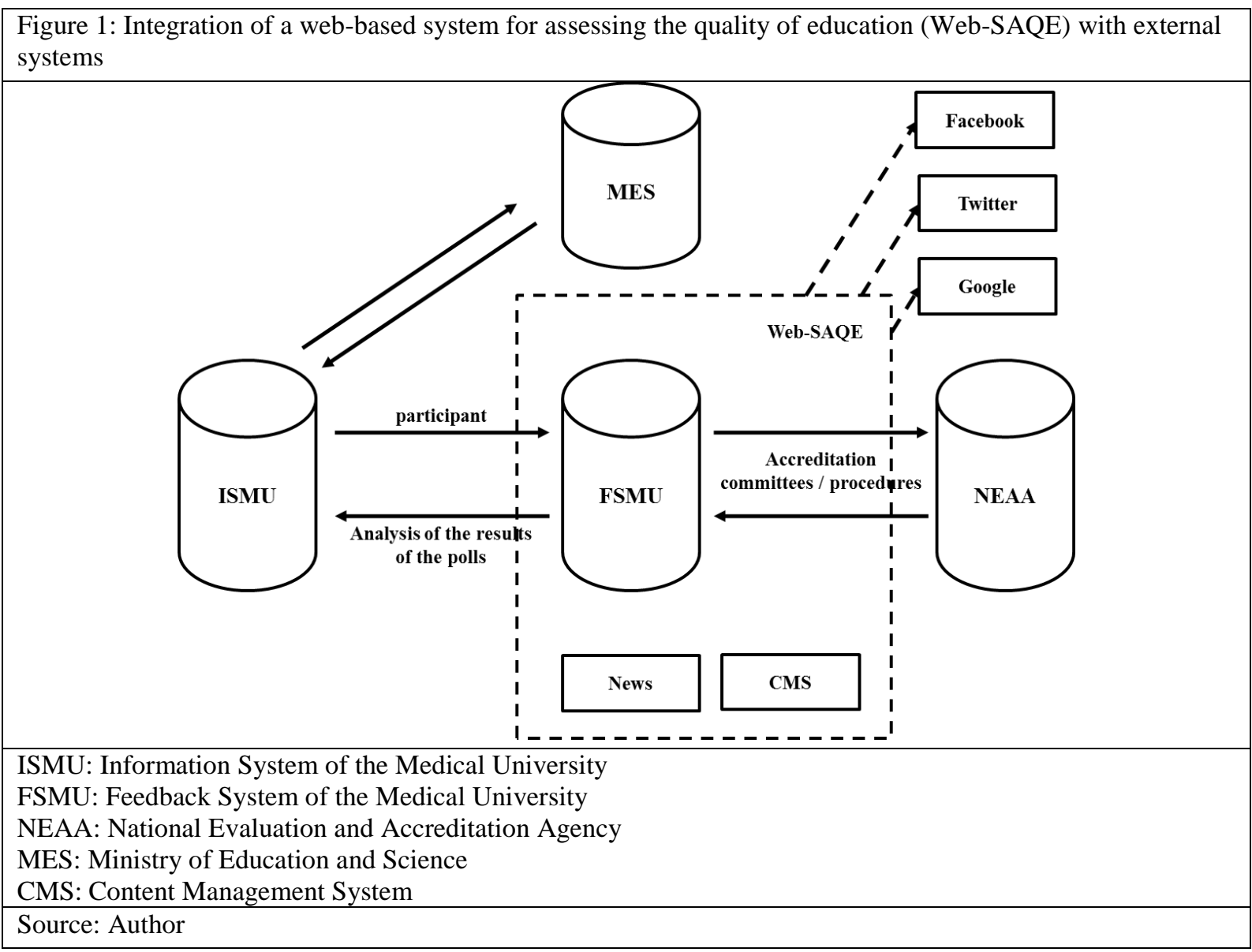

Figure 2 shows the software architecture of feedback system of the medical university integrated with the information system of the medical university.

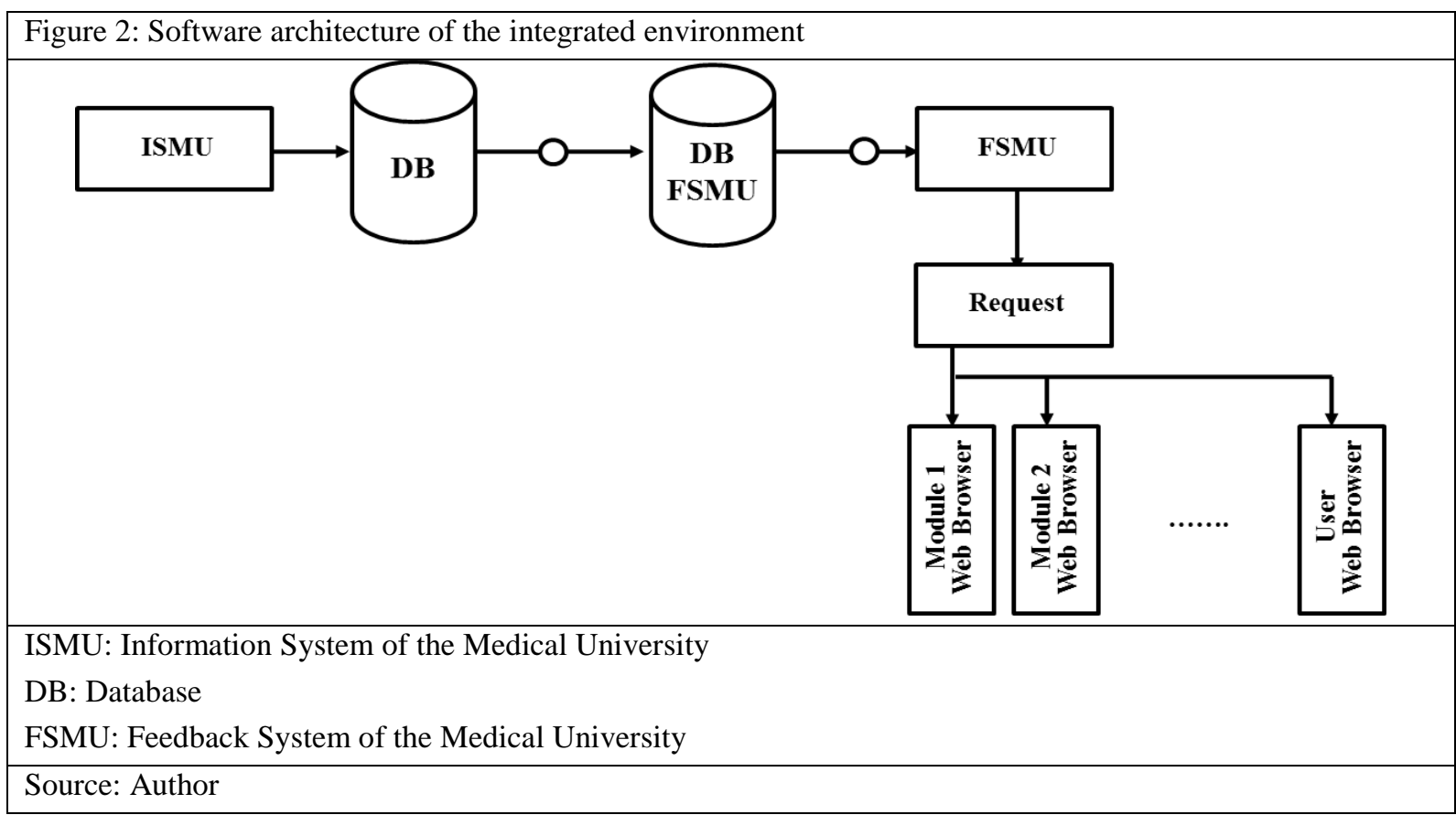

Figure 2 shows that the functional levels of building the architecture are the web-client, web-server, application server, server database, and requests.

Figure 3 illustrates the different elements in managing the feedback system of the medical university. These elements are subject to design and future implementation. 


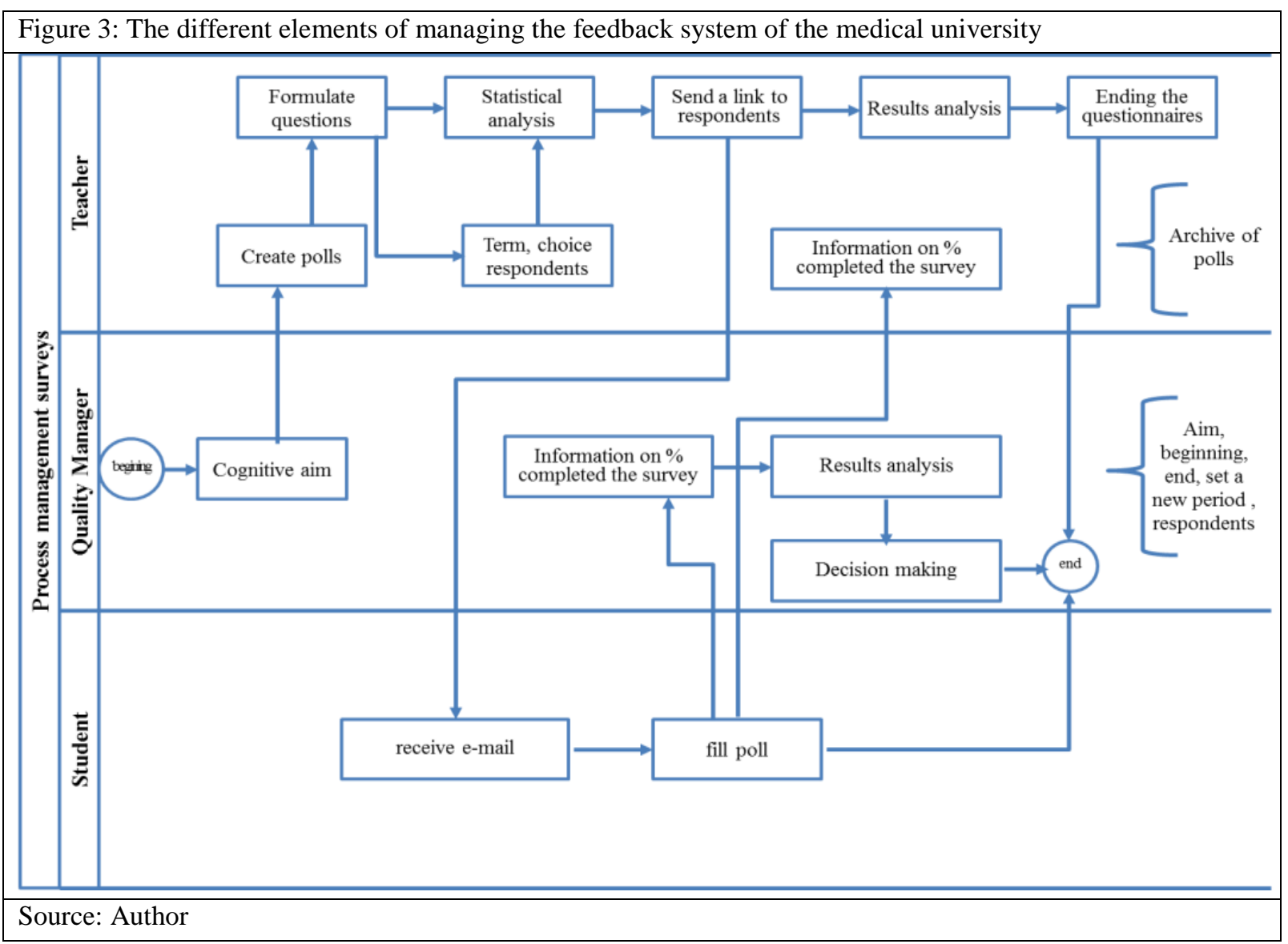

The module for monitoring user activity involves realization of a role model in the system for identification and authorization. Figure 4 shows the authorization process.

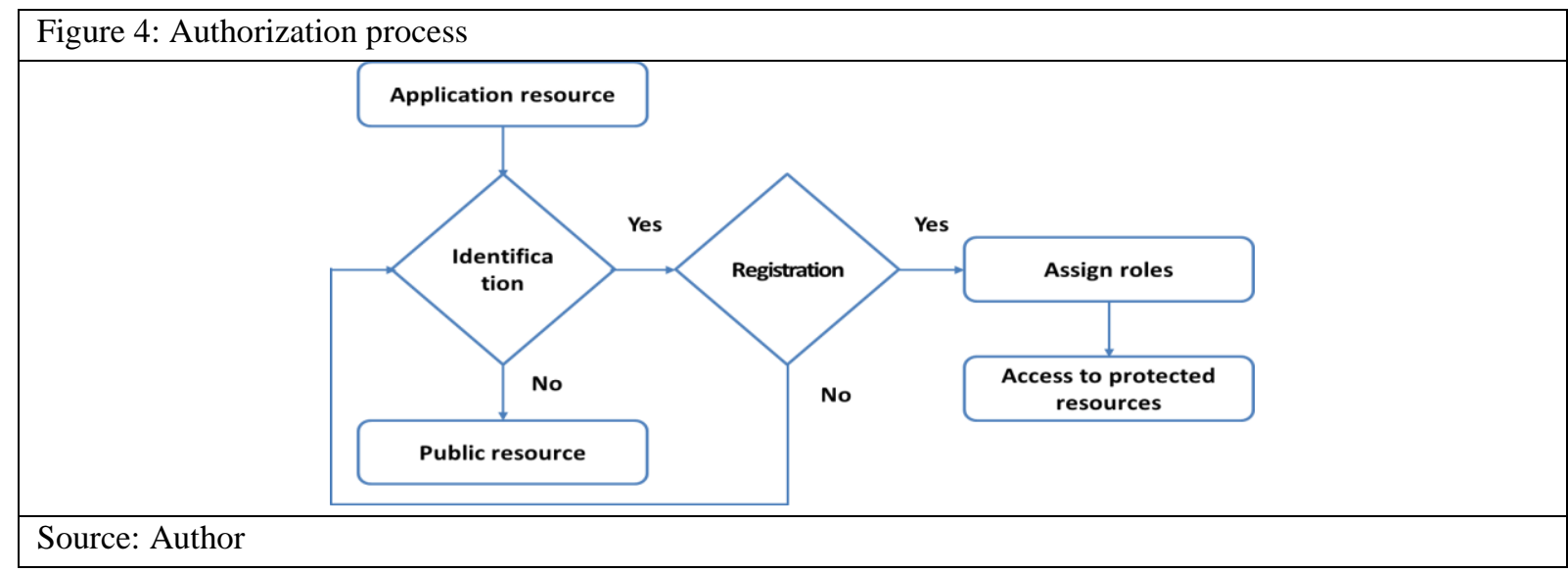

When the registration is successfully completed, the Web-SAQE sends an e-mail advising this. The user is able to restore their forgotten password. As well, a teacher can also act as an administrator or a manager.

Providing personalized access to a designed software system highlights the issue of protecting data of these services and restricting access to information. For creating access restrictions at a functional level, this group of services must meet the following requirements:

- The student has the right to manage their right to freedom of opinion. If it is already exercised, he or she only sees how the respondents have voted without having access to the analyzed outcomes.

- The teacher sees the percentage of the respondents who have voted at the time. He or she creates, edits and deletes the questions in the survey. If the survey is $100 \%$ completed, the teacher is able to end the form completion before the deadline. 
- The manager or supervisor defines the cognitive aim and the management; he or she identifies those who are responsible for the survey and sets the duration of the survey; he or she is informed about the process of conducting the survey and then of its results.

Creating restrictions at a database level are performed by

- administrator of the system;

- teacher; and

- administrator of the survey.

These requirements provide two levels of information security: functional security and data security. Functional security involves determining user access to system functions. Data security includes providing user access to the specific data.

The information system provides services for user management and access control to information. The system allows access in two ways: 1) through public (anonymous) access, and 2) through identification and authorization.

Regarding approaches in the design of software architecture, modern paradigms for designing the architecture of the software application offered by Bontchev (2012) are object-oriented design, component-oriented design, design based on a contract and context-oriented programming, and service-oriented architecture design.

The object-oriented design is based on creating classes as a container of objects. Component-based architecture and service-oriented architecture are currently at the center of the object-oriented design. The component-oriented design software introduces a new paradigm in which systems do not work from scratch, but rather involve a cluster of existing components (Berberova \& Bontchev, 2009). Their construction aims to reduce the cost of the assembly through prototyping rapid-built software systems that are flexible, lightweight and easily maintained.

The design, based on a contract and context-oriented programming, requires software designers to define the interface specification of the software components precisely. A major part of this methodology is the question of how the elements cooperate with each other.

Designing the architecture of the Web-SAQE involves the component-oriented paradigm (Figure 5). This is due to the large range of complete technology solutions that currently exist.

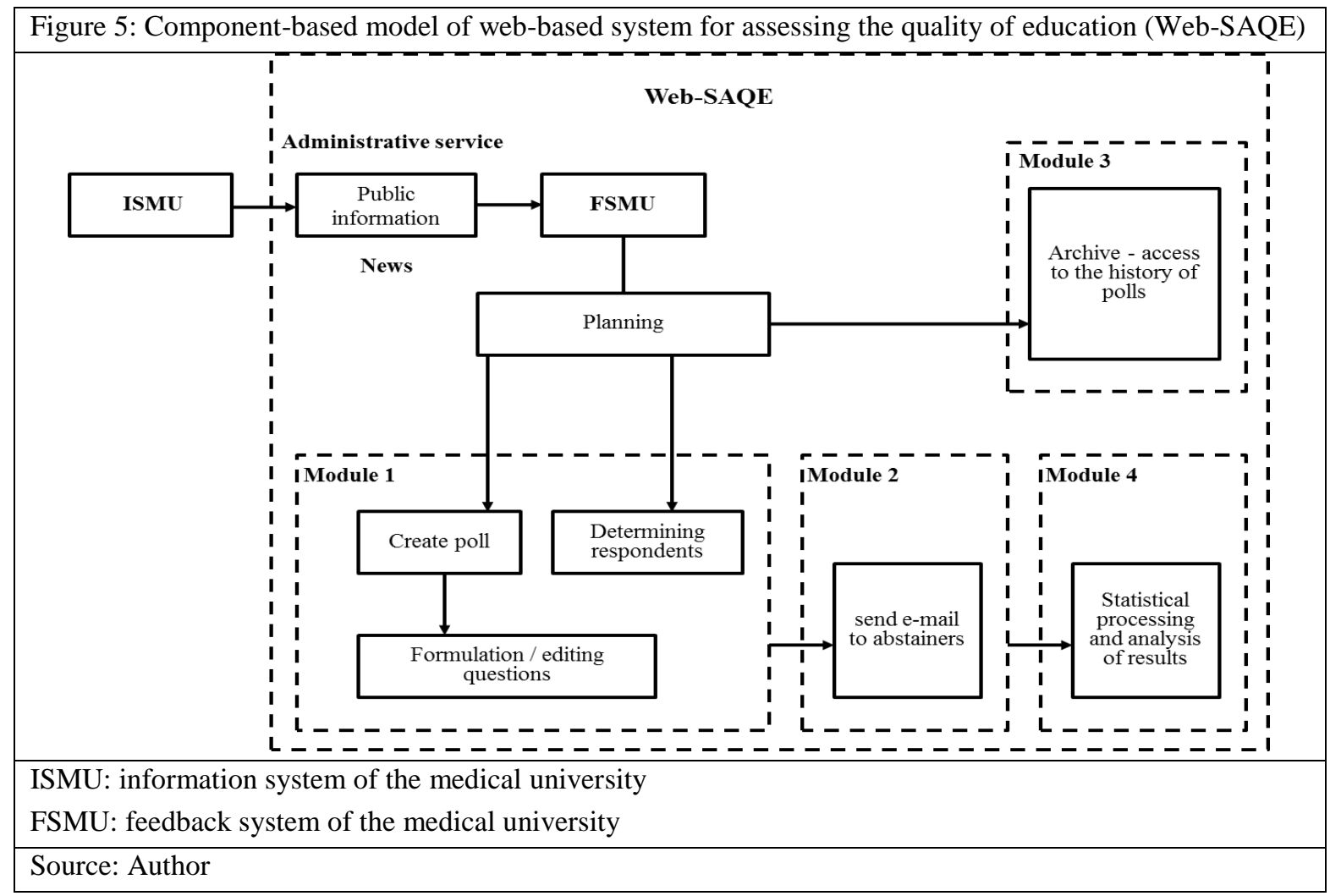


The design of the individual components is governed by two main principles in software design: poor connectivity and strong consistency. The less information exchanged between the components (i.e., components are simpler), the weaker will be the connection. The main task for consistency is for all elements inside the component to solve a common problem (Stoyanov, Stoyanova, \& Doychev, 2006).

\section{Conclusions}

The presented model of the feedback system of the medical university as a component of the WebSAQE complies with specific requirements for compatibility with the university information system and forms part of it. The Web-SAQE is designed to operate as an integrated Web-based system. It provides a high level of security and safety of the data (in data transmission so that they cannot be intercepted or modified) by the creation of backups, archiving, recovery, and protection. A high level of data integration is maintained by providing a repository for data and system development for updating the data, suggesting the use of an integrated and dynamic database. The Web-SAQE provides access to all pre-authorized users (students, teachers, and employees) regardless of their location. The system is compatible to work with mobile devices (smartphones, tablets, and netbooks). The architecture of the system is based on a modular principle for easy integration into various functional modules. Finally, the survey module, which is incorporated in the Web-SAQE, provides the ability to manage surveys dynamically.

\section{References}

Berberova, D., \& Bontchev, B. (2009). Design of Service Level Agreements for Software Services. In Proceedings of the International Conference on Computer Systems and Technologies and Workshop for PhD Students in Computing (pp. 277282). inproceedings, New York, NY, USA: ACM. https://doi.org/10.1145/1731740.1731769

Bontchev, B. (2012). Design of software tools and platforms for technology enhanced learning Habilitation thesis for promotion to Full Professor. Sofia University Saint Kliment Ohridski.

Debuse, J., Lawley, M., \& Shibl, R. (2007). The Implementation of an Automated Assessment Feedback and Quality Assurance System for ICT Courses. Journal of Information Systems Education, 18(4), 491-502.

Doneva, R., Kasakliev, N., \& Lazarova, V. (2011). Towards an Integrated Information System of Secondary Education. In National Conference "Education in the Information Society" (pp. 149-156). Plovdiv.

Hadzhikoleva, S., Hadzhikolev, E., Doneva, R., \& Totkov, G. (2010). Web-based system for quality assessment of elearning in higher education. https://doi.org/10.13140/RG.2.1.2927.7686

Kilova, K., \& Stoyanova, R. (2016). Student's Opinion on the Use of Information System for Assessing and Monitoring the Quality of Education at the Medical University - Plovdiv. International Journal of Knowledge. Knowledge in Practice, 15(2), 849-852.

Lazarova, V. (2015). Integrated Web-based information system for secondary education. PU "Paisiy Hilendarski” Plovdiv.

Peeva, K. (2010). Theoretical Aspects of the Assessment of Quality in Higher Education. Trakia Journal of Sciences, 8 (2), $429-435$.

Stoyanov, S., Stoyanova, A., \& Doychev, E. (2006). Software technologies. Plovdiv.: University Publishing "Paisiy Hilendarski." 